\title{
Whole body MRI for Breast Cancer Staging: the roles of the Field Strength and Gadolinium Contrast Agents in Focus
}

\author{
Daniel Wiech' ${ }^{1}$, Stefanie Peix ${ }^{1}$, Dorothee Predel ${ }^{1}$, Claudia Kurrat ${ }^{1}$, Joachim Feger ${ }^{1}$, Arnhild Kott ${ }^{1}$, Marios-Nikos \\ Psychogios $^{2}$, Stathis Hadjidemetriou ${ }^{3}$, Ansgar Malich ${ }^{1}$ and Ismini E Papageorgiou ${ }^{1,4^{\star}}$ \\ Institute of Radiology, Südharz Hospital Nordhausen, Nordhausen, Germany \\ 2Institute for Diagnostic and Interventional Neuroradiology, University Hospital of Goettingen, Goettingen, Germany \\ ${ }^{3}$ Department of Electrical Engineering and Informatics, Cyprus University of Technology, Limassol, Cyprus \\ 4Institute of Diagnostic and Interventional Radiology, University Hospital of Jena, Jena, Germany
}

\section{Article Info}

*Corresponding author:
Ismini E Papageorgiou
Institute of Diagnostic and Interventional
Radiology
University Hospital of Jena, Jena
Institute of Radiology
Südharz Hospital Nordhausen, Nordhausen
Germany
E-mail: ismini.e.papageorgiou@shk-ndh.de

Received: April 14, 2018

Accepted: May 2, 2018

Published: May 7, 2018

Citation: Wiech D, Peix S, Predel D, et al. Whole body MRI for Breast Cancer Staging: The roles of the Field Strength and Gadolinium Contrast Agents in Focus. Madridge J Oncogenesis. 2018; 2(1): 35-40. doi: 10.18689/mjo-1000106

Copyright: (c) 2018 The Author(s). This work is licensed under a Creative Commons Attribution 4.0 International License, which permits unrestricted use, distribution, and reproduction in any medium, provided the original work is properly cited.

Published by Madridge Publishers

\begin{abstract}
Purpose: Whole-body magnetic resonance imaging (WB-MRI) is emerging as a powerful diagnostic tool for breast cancer staging, especially for the detection of bone metastasis. However there is a low level of evidence concerning the added benefit from the use of higher field strengths and contrast agents. In this study we compare the diagnostic efficacy of WB-MRI for the field strengths $1.5 \mathrm{~T}$ and $3 \mathrm{~T}$ commonly used in the clinic. Moreover, we investigate the added benefit from gadolinium contrast agent application towards the effective detection of breast cancer bone metastasis.
\end{abstract}

Methods and Materials: The study was retrospective for 855 patients scanned between 05/2007 and 08/2017. 345 patients were imaged at $1.5 \mathrm{~T}$ and 510 at 3T field strength (Philips Achieva or Ingenia) with a T1w FFE and a STIR or a Dixon at the coronal orientation. In 431 patients we injected gadoteridol (ProHance ${ }^{\circledR}$ ) $0.1 \mathrm{mmol} / \mathrm{kg}$. Clinical confirmation with skeletal scintigraphy or bioptic confirmation served as the ground truth.

Results: The sensitivity (SE) and specificity (SPE) for $1.5 \mathrm{~T}$ were $98.34 \% / 91.24 \%$ and the positive predictive value (PPV)/negative predictive value (NPV) were $86.03 \% / 99 \%$. A field strength of 3 T showed SE/SPE of $100 \% / 92.81 \%$ and PPV/NPV of $83.22 \% / 100 \%$. Binary logistic regression with Fisher's exact test revealed no significant difference between 1.5T and 3T WB-MRI ( $P$ 0.663, odds ratio 0.839). The SE/SPE of WB-MRI (merged 1.5T and 3T) without enhancement were $98.66 \%$ 91/60\%, with PPV/NPV 86.54\%/99.20\%. Upon administration of contrast agent, the SE/SPE shifted to $100 \% / 92.83 \%$ and the PPV/NPV to $82.70 \% / 100 \%$. Binary logistic regression with Fisher's exact test returned no significant effect for the contrast agent ( $P 0.836$, odds ratio 0.9$)$.

Conclusion: WB-MRI is a highly specific and sensitive diagnostic tool for bone metastasis in breast cancer with equal diagnostic efficacy in the field strengths of $1.5 \mathrm{~T}$ or $3 \mathrm{~T}$. Gadolinium can be omitted without affecting the diagnostic accuracy and its usage should be spared only for problem-solving.

Keywords: Breast cancer; Staging; Gadoteridol; Dixon; Bone scan; Pregnancy.

Abbreviations: 1.5T: 1.5 Tesla; 3T: 3.0 Tesla; 18F-FDG PET/CT: 2-deoxy-2-[fluorine-18] fluoro- D-glucose integrated with computed tomography; ACR: American College of Radiology; Cl: Confidence Interval; CT-CAP: Computed Tomography of Chest - Abdomen - Pelvis; ESMO: European Society for Medical Oncology; FFE: Fast Field Echo; MRI: 
Magnetic Resonance Imaging; NCCP: National Cancer Control Programme; NICE: National Institute for Health and Care Excellence; NPV: Negative Predictive Value; PPV: Positive Predictive Value; SE: Sensitivity; SNR: Signal to Noise Ratio; SPE: Specificity; STIR: Short Tau Inversion Recovery; T1w: T1 Weighted Imaging; T2w: T2 Weighted Imaging; TSE: Time Spin Echo; VIBE: Volumetric Interpolated Breath-hold Examination; WB-MRI: Whole Body MRI.

\section{Introduction}

Whole-Body Magnetic Resonance Imaging (WB-MRI) as a diagnostic tool for the detection of bone metastases is a developing technological achievement of the past decade. A systematic review and meta-analysis of 11 studies conducted in 2011 estimated a pooled sensitivity (SE) of $90 \%$ with a $95 \%$ Confidence Interval (Cl) of $84-94 \%$ and a specificity (SPE) of $92 \%$, with a $95 \% \mathrm{Cl}$ of $88-95 \%$ for the detection of osseous metastatic disease [1]. With this performance WB-MRI is the second more sensitive and specific method for the detection of bone metastasis after the 2-deoxy-2-[fluorine-18] fluoro-Dglucose PET integrated with computed tomography (18F-FDG $\mathrm{PET} / \mathrm{CT}$ ) and a current trend in breast cancer staging, which is steadily gaining ground due to the lack of ionizing radiation, excellent soft tissue contrast, lower cost and increased availability over 18F-FDG PET/CT [2,3]. The lack of ionizing radiation makes WB-MRI the method of choice for pregnant women [4].

In the current guideline recommendations for bone metastatic disease in breast cancer, the National Cancer Control Programme (NCCP) suggests a Computed Tomography of Chest-Abdomen-Pelvis (CT-CAP) and whole body isotope scan for the staging of newly diagnosed breast cancer (Grade B evidence in the v.7, valid as of 2015). A PET/CT is recommended in only special cases and not as mainstream imaging modality (Grade C evidence [5]), although some studies suggest PET/CT as a fist-line diagnostic tool due to its high accuracy [6]. The American College of Radiology (ACR) Appropriateness Criteria ${ }^{\circledR}$ as revised in 2016 recommend a whole body 18 F-FDG PET/CT scan or a Tc-99m whole body scan to rule out bone metastases in newly diagnosed cancer and Tc-99m bone scans in surveillance [7-9]. The National Institute for Health and Care Excellence (NICE) in the guidelines of 2017 recommends equivocally CTCAP, MRI or Tc- $99 \mathrm{~m}$ whole body scan for the exclusion of metastatic disease in the axial skeleton [10]. Finally, the recent European Society for Medical Oncology (ESMO) guidelines recommend a CT-CAP or Tc-99m body scan for breast cancer skeletal staging without mentioning WB-MRI [11].

Despite the fact that WB-MRI is not widely included in the recent guidelines for breast cancer staging, recent studies converge towards a possibly beneficial role compared to CTCAP $[12,13]$. WB-MRI is implemented as an early screening tool in women with breast cancer in our department since 2007 using the most widely available field strengths in the clinic of $1.5 \mathrm{~T}$ and $3 \mathrm{~T}$. In the current study we aim to (i) retrospectively assess the diagnostic accuracy of WB-MRI for the detection of bone metastatic disease in breast cancer and (ii) compare the effectiveness of different field strengths.
Moreover, (iii) we questioned the necessity of gadolinium contrast agent by comparing the diagnostic accuracy of nonenhanced sequences (T1W and STIR) to the contrasted fatsuppressed Dixon. We provide evidence that WB-MRI with field strength of 1.5T or 3T can be used for the diagnosis of bone metastasis in breast cancer with equal diagnostic efficacy and independently of the application of gadolinium.

\section{Methods}

\section{Recruitment and Flow of Participants}

The study is retrospective for breast cancer patients screened with WB-MRI for bone metastasis in Südharz Klinikum Nordhausen between 2007 and 2017 and was performed in accordance with the STARD guidelines $[14,15]$. Due to the retrospective character of the study, the ethical committee of the University of Jena has waived the mandate for a written patient informed consent. From a total of 1119 investigated patients, 264 patients were excluded from further analysis due to lack of any form of ground truth (biopsy or follow up data). No other eligibility criteria applied. From the total of 855 eligible patients (58 \pm 11 y.o.), 510 (134 with bone metastases) were scanned with a $3 \mathrm{~T}$ field strength setup (Philips Ingenia, Philips Medical Systems, Böblingen, Germany) and 345 (126 with bone metastases) with a 1.5T MRI system (Philips Achieva or Philips Ingenia, Philips Medical Systems). The field strength selection was solely determined by the patient compatibility with a $3 \mathrm{~T}$ static magnetic field and scanner availability. The incompatibility with $3 \mathrm{~T}$ field strength was mainly determined through medical implants with manufacturer-defined safety up to $1.5 \mathrm{~T}$. Subjects with tattoos larger than $15 \mathrm{~cm}$ or permanent make-up were preferentially investigated at $1.5 \mathrm{~T}$ due to susceptibility to skin burns at high Specific Absoption Rate (SAR) values. No age or disease criteria applied. In 431 patients (110 with bone metastases) the WB-MRI was contrasted with gadoteridol (ProHance ${ }^{\circledR}$, Bracco Imaging S.p.A., Konstanz, Germany) $0.1 \mathrm{mmol} / \mathrm{kg}$, whereas 424 patients (150 with bone metastases) either did not consent for gadolinium application or had a medical contraindication such as a contrast agent allergy or a deteriorated renal function. In these cases the contrast agent was omitted (Figure 1).

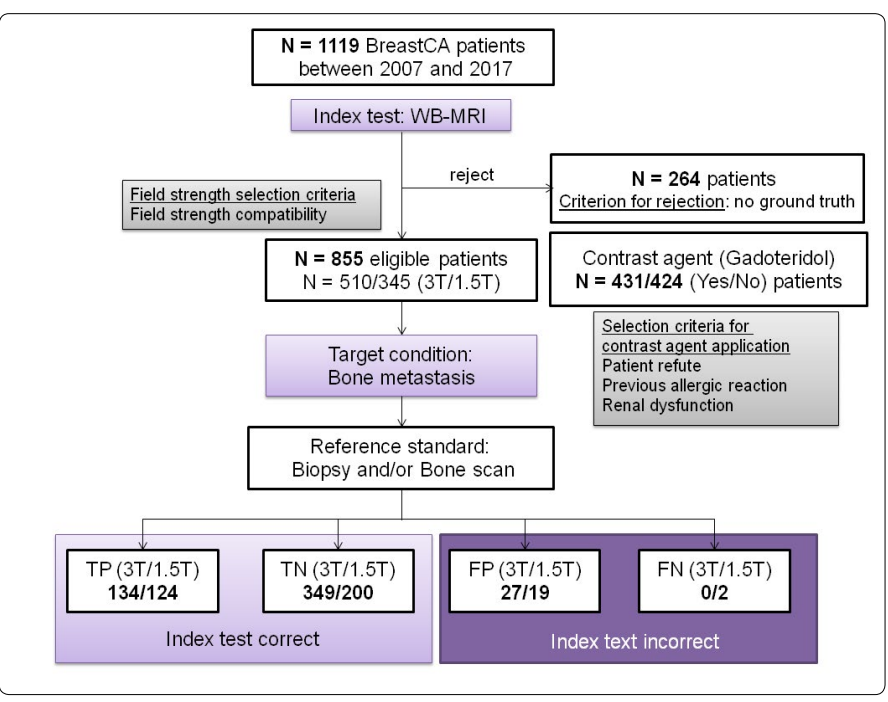

Figure 1. STARD diagram. Flow of participants 


\section{Imaging and Image Evaluation}

WB-MRI images were acquired with an 108-channel dStream Whole-Body coil (Philips Medical Systems) (Figure 2 $A$ and $B$ ) which combines two Flex Coverage Anterior coils with a Flex Coverage Posterior, Head Neck and Base coil to enable a total coverage of $200 \mathrm{~cm}$. The protocol consisted of the following coronal sequences in brief: (i) a T1weighted (T1w) sequence in Time Spin Echo (TSE) or Fast Field Echo (FFE) technique, (ii) a Short Tau Inversion Recovery (STIR) and (iii) a 3D T1w FFE sequence for the Dixon technique with gadoteridol contrast as previously referred (Figure $2 \mathrm{C}$ ).
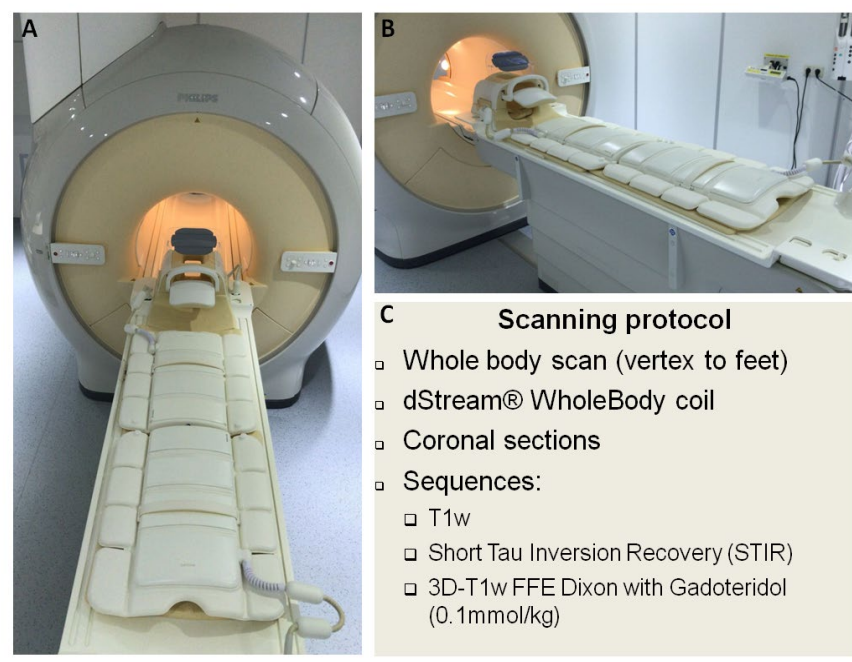

- Whole body scan (vertex to feet) dStream ${ }^{\circledR}$ WholeBody coil

Coronal sections

Sequences:

$\square \mathrm{T} 1 \mathrm{w}$

口 Short Tau Inversion Recovery (STIR)

a 3D-T1wFFE Dixon with Gadoteridol $(0.1 \mathrm{mmol} / \mathrm{kg})$

Figure 2. All scans took place in Philips MRI scanners 1.5T and 3T using a dStream whole-body coil (A and $B)$. The scanning protocol is summarized in (C)

Protocol details are summarized in Table 1 and Table 2, respectively.

Table 1. Whole Body MRI technical factors for the $1.5 \mathrm{~T}$ scanner (Philips Ingenia)

\begin{tabular}{|c|c|c|c|}
\hline & T1 TSE & STIR & 3D T1 Dixon FFE \\
\hline Patient position & \multicolumn{3}{|c|}{ Supine, arms down at sides, head first } \\
\hline Imaging plane & \multicolumn{3}{|c|}{ Coronal } \\
\hline Coil(s) & \multicolumn{3}{|c|}{ dStream Whole-Body Coil (Philips) } \\
\hline Contrast & No & No & Gadoteridol $0.1 \mathrm{mmol} / \mathrm{kg}$ \\
\hline Anatomic coverage & \multicolumn{3}{|c|}{ Cranial vertex to feet } \\
\hline TE ms & 4 & 70 & TE1 1.69 TE2 3.7 \\
\hline TR ms & 685 & 6839 & 5.4 \\
\hline TI (IR delay) & & 150 & \\
\hline Flip angle $\circ$ & 90 & 130 & 15 \\
\hline Echo train length & 11 & & \\
\hline Number of slices & 66 & 66 & 115 \\
\hline $\begin{array}{l}\text { ACQ voxel size } \\
\left(F H^{\star} R L^{*} A P\right) \mathrm{mm}\end{array}$ & $1.6 * 2 * 3$ & $2 * 2 * 3$ & $2 * 2 * 4$ \\
\hline $\begin{array}{l}\text { Recon voxel size } \\
\left(\mathrm{FH}{ }^{\star} \mathrm{RL}\right) \mathrm{mm}\end{array}$ & $1.17 * 1.17$ & $0.95 * 0.95$ & $0.94 * 0.94$ \\
\hline Slice thickness $\mathrm{mm}$ & 3 & 3 & 2 \\
\hline Spacing (slice gap) mm & 0.3 & 0.3 & 0.2 \\
\hline $\mathrm{FOV}\left(\mathrm{FH}{ }^{\star} \mathrm{RL}{ }^{*} \mathrm{AP}\right) \mathrm{mm}$ & $300 * 450 * 217.5$ & $300 * 455.7 * 217.5$ & $450 * 450 * 230$ \\
\hline $\begin{array}{c}\text { Matrix } \\
\text { (phase*frequency) }\end{array}$ & $215 * 188$ & $226 * 152$ & $224 * 224$ \\
\hline Acquisition time (min) & 7.55 & 5.30 & 1.19 \\
\hline
\end{tabular}

Table 2. Whole Body MRI technical factors for the 3T scanner (Philips Ingenia)

\begin{tabular}{|c|c|c|c|}
\hline & T1 FFE & STIR & 3D T1 Dixon FFE \\
\hline Patient position & \multicolumn{3}{|c|}{ Supine, arms down at sides, head first } \\
\hline Imaging plane & \multicolumn{3}{|c|}{ Coronal } \\
\hline Coil(s) & \multicolumn{3}{|c|}{ dStream Whole-Body Coil (Philips) } \\
\hline Contrast & No & No & \\
\hline Anatomic coverage & \multicolumn{3}{|c|}{ Cranial vertex to feet } \\
\hline TE ms & 2.4 & 60 & TE1 1.32. TE2 2.3 \\
\hline TR ms & 91 & 11340 & 3.6 \\
\hline TI (IR delay) ms & & 200 & \\
\hline Flip angle ${ }^{\circ}$ & 70 & 70 & 10 \\
\hline \multicolumn{4}{|l|}{ Echo train length } \\
\hline Number of slices & 60 & 60 & 115 \\
\hline $\begin{array}{l}\text { ACQ voxel size } \\
\left(F H^{*} R L^{*} A P\right) m m\end{array}$ & $1.47 * 2.14 * 3$ & $1.8 * 2.44 * 3$ & $1.5^{\star} 1.5^{\star} 4$ \\
\hline $\begin{array}{l}\text { Recon voxel size } \\
\left(\mathrm{FH} H^{*} \mathrm{RL}\right) \mathrm{mm}\end{array}$ & $1.02 * 1.02$ & $1.04 * 1.04$ & $0.94 * 0.94$ \\
\hline Slice thickness $\mathrm{mm}$ & 3 & 3 & 2 \\
\hline Spacing (slice gap) & 1 & 1 & 2 \\
\hline $\mathrm{FOV}\left(\mathrm{FH} \mathrm{HL}^{*} \mathrm{AP}\right) \mathrm{mm}$ & $263 * 493 * 239$ & $263 * 499.25 * 239$ & $300 * 450 * 240$ \\
\hline $\begin{array}{c}\text { Matrix } \\
\text { (phase*frequency) }\end{array}$ & $233 * 180$ & $151 * 148$ & $300 * 200$ \\
\hline Acquisition time (min) & 3.51 & 9.04 & 2.09 \\
\hline
\end{tabular}

All images were evaluated by 2 radiologists, one with low (2 years) or intermediate (5 years) experience and a consultant with more than 15 years of experience (AM, AK, CK, JF and $D P)$. The ground truth of metastatic disease was confirmed either with a Tc-99m bone scan or with a bone biopsy.

\section{Statistics and Software}

Logistics and descriptive statistical data processing were performed with Libre Office ${ }^{\mathrm{TM}}$ 4.4.7.2 (The Document Foundation, Berlin, Germany) and the Microsoft@) Office suite 2010 (Microsoft Ireland Operations Limited, Dublin, Ireland). Graphical processing was accomplished using the free source platform Inkscape (License name: GPL v2+, https://inkscape.org). Percentages are rounded up to the second decimal place.

\section{Results}

WB-MRI for breast cancer staging was applied in different magnetic field strengths, $3 \mathrm{~T}$ and 1.5T, with single selection criterion the patients' compatibility with higher field strengths. The imaging protocol in both devices includes coronal T1w scans vertically stitched to a whole-body image as displayed in Figure $3 \mathrm{~A}$ and Figure $3 \mathrm{D}$ for 1.5T and 3T, respectively. The T1w sequences outline the tissue anatomy with a voxel analysis of ca. $1.5 * 2 * 3 \mathrm{~mm}(x, y, z)$ in both field strengths (see Tables 1 and 2 for technical details). The lower resolution, fat-suppressed STIR sequences (Figure $3 B, C E, F$ ) intensify the signal in regions where the fatty bone marrow is replaced by metastatic cells and extravasated fluid. Examples of osseous metastasis in the lumbar spine (Figure $3 \mathrm{~B}, \mathrm{E}$ ) and in the left femur head (Figure $3 \mathrm{C}, \mathrm{F}$ ) as depicted in 1.5T (Figure $3 \mathrm{~B}, \mathrm{C}$ ) and $3 \mathrm{~T}$ field strength (Figure $3 \mathrm{E}, \mathrm{F}$ ) are annotated with white arrows.

The primary aim of this study has been to evaluate the diagnostic accuracy of WB-MRI for the detection of osseous metastasis in breast cancer. 1.5T scanners have higher availability, 
lower costs of obtaining and maintenance and better compatibility with medical implants compared to 3T or even higher field scanners, however at the cost of lower image resolution due to a reduced signal-to-noise-ratio (SNR) [16-18]. We examined whether the field strength indeed influences the diagnostic accuracy (Figure 3D). Both field strengths were retrospectively evaluated with a high sensitivity/specificity (SE/SPE) of 98/91\% and 100/93\% for $1.5 T$ and $3 \mathrm{~T}$, respectively. The positive predictive value (PPV) for $1.5 \mathrm{~T}$ was $86 \%$ and for $3 \mathrm{~T}$ was $83 \%$, whereas the negative predictive value (NPV) reached $99 \%$ and $100 \%$ for $1.5 T$ and 3 T (Figure 3 D), respectively. Despite the slight difference in disease prevalence among the tested populations, which can affect the PPV and the NPV [19], Fischer's exact test showed a statistically equivalent diagnostic performance for both scanners (P 0.663, Figure $3 \mathrm{D})$.

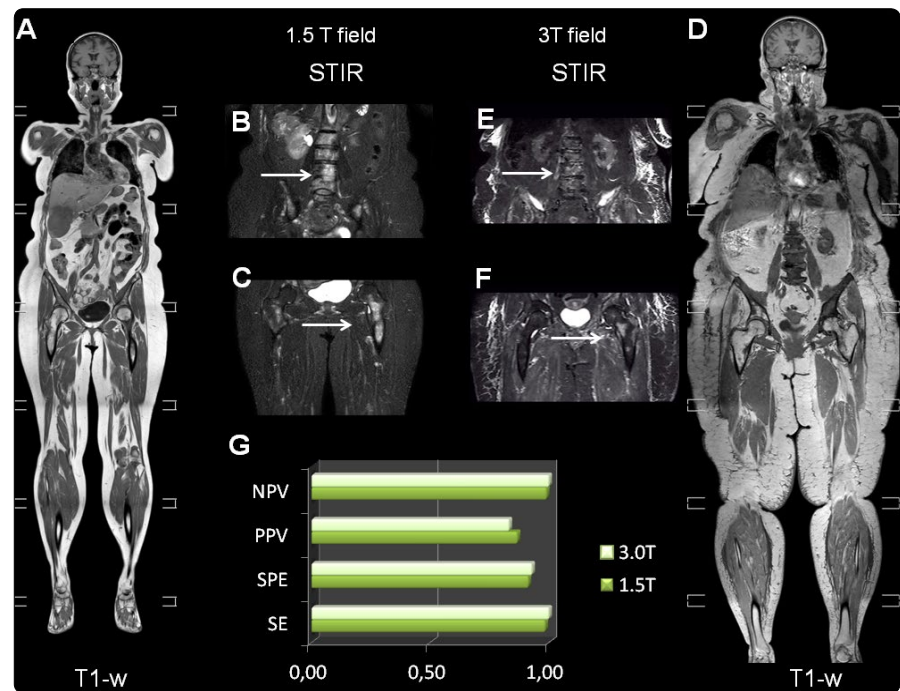

Figure 3. Equal WB-MRI diagnostic accuracy for 1.5T (A-C) and 3T (D-F) field strength. T1w sequences in whole-body stitched reconstruction (A) 1.5T and (D) 3T. (B, E) Lumbar spine bone metastases and (C, F) left femur neck metastatic disease (white arrows) as depicted using STIR in different field strengths, $(G)$ Diagnostic accuracy for 1.5T (dark green, $\mathrm{N}=345$ ) and 3T (light green, $N=510$ ). SE, sensitivity; SPE, specificity; PPV, positive predictive value; NPV, negative predictive value. Binary logistic regression with Fischer's exact test, $P$ 0.663.

Ca. 50\% (431 from 855 eligible patients) received gadoteridol and the others were investigated without a contrast agent due to intolerance, allergic reaction or renal dysfunction. In Figure 4, example WB-MRI images illustrate the typical appearance of the osseous metastatic disease as high-intensity bone lesions in fat-suppressed sequences without (Figure 4 B, STIR) and with (Figure 4 D, T1w Dixon) contrast agent. Osseous metastases are located in the bone marrow and induce intensified spots on STIR images due to bone marrow edema as well as a vivid contrast agent accumulation in the contrasted Dixon sequences due to neo angiogenesis (Figure $4 \mathrm{~B}$ and $\mathrm{D}$, white arrows for STIR and Dixon, respectively) [2,20]. Harmonizing to the current advances on gadolinium safety in the clinical routine [21-23], we questioned whether the patient group that received contrast agent experienced an objective diagnostic benefit (Figure $4 \mathrm{E}$ ). Since both field strengths displayed equal diagnostic value, data from both the $1.5 \mathrm{~T}$ and the $3 \mathrm{~T}$ scanner were merged for this evaluation. The SE/SPE for the non-contrasted and contrasted group was approximately 99/92\% and 100/93\%, respectively. The PPV was ca. $87 \%$ without and $83 \%$ with gadolinium and the NPV was $99 \%$ and $100 \%$ without and with contrast agent, respectively. Fischer's exact test provides no significant difference ( $P$ 0.836), which implies that the diagnostic accuracy is independent of the gadolinium application (Figure $4 \mathrm{E}$ ).

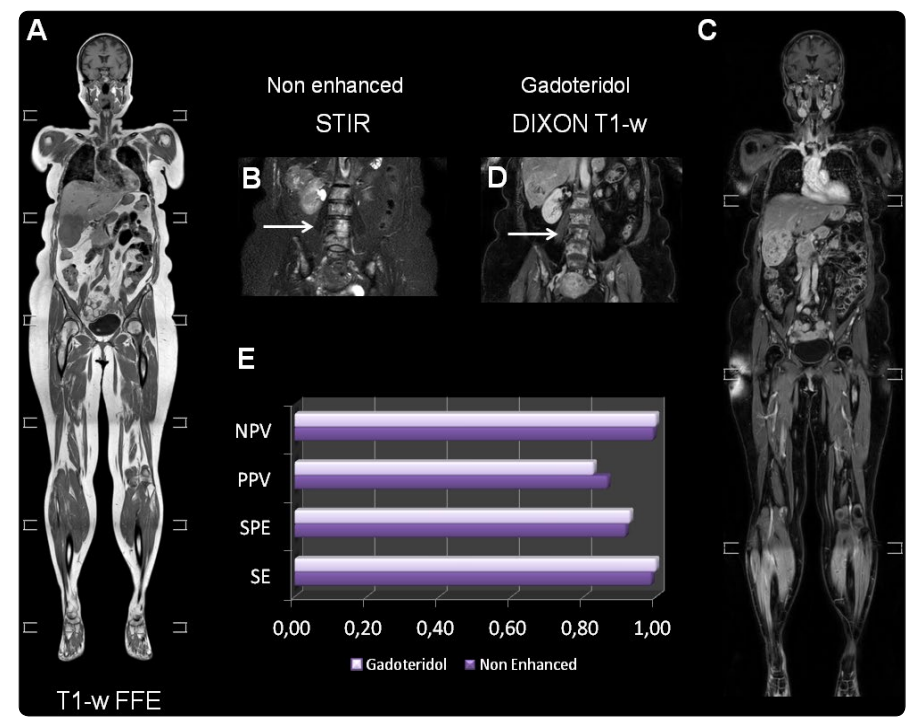

Figure 4. The WB-MRI diagnostic accuracy is not influenced by gadolinium. $(A, B) 1.5 T$ without contrast, T1w and STIR, $(C, D) 1.5 T$ with additional contrasted Dixon sequence (gadoteridol $0.1 \mathrm{mmol} /$ $\mathrm{kg}$ ). The whole-body stitched T1w images are without ( $\mathrm{A}$, left) and with ( $C$, right) contrast agent. Lumbar spine bone metastases (white arrows) as depicted using STIR (B) and contrasted Dixon (D). (E)

Diagnostic accuracy of WB-MRI without contrast agent (non enhanced, deep purple, $N=431$ ) and contrasted with gadoteridol $0.1 \mathrm{mmol} / \mathrm{kg}$ (light purple, $\mathrm{N}=424$ patients). SE, sensitivity; SPE, specificity; PPV, positive predictive value; NPV, negative predictive value. Binary logistic regression with Fischer's exact test, $P 0.836$.

Summarizing the above, we conclude that WB-MRI is an accurate diagnostic method for the detection of bone metastases in breast cancer. Field strengths of $1.5 \mathrm{~T}$ or $3 \mathrm{~T}$ reveal a statistically equivalent diagnostic efficacy, which is further not significantly improved by the use of gadolinium contrast agent.

\section{Discussion}

In this study we assess the diagnostic accuracy of WBMRI for the detection of bone metastases in a sample of 855 patients focusing on the roles of the field strength and the potential benefit from gadolinium contrast application.

Several studies support WB-MRI as a cost-effective, widely available and accurate method for the detection of bone metastases of solid tumors and for multiple myeloma, which can modify the diagnostic decisions compared to other diagnostic methods such as CT and bone scan [12]. The diagnostic accuracy of STIR sequences has been evaluated as early as 2000 with a high sensitivity in the range of $96 \%$ for the osseous metastatic disease [24] and is currently a standard sequence for WB-MRI imaging protocols $[12,25,26]$. Thomson et al. have tested the efficacy of the 3D Volumetric Interpolated Breath-hold Examination (VIBE) with subtraction between the contrasted and the non contrasted images as an alternative with promising results [27]. Implementation of the Dixon technique for WB-MRI provides a high resolution diagnostic battery. Costelloe et al. compared the metastatic bone lesion conspicuity in contrasted 
Dixon and STIR, reporting a significant advantage of the contrasted Dixon [28]. Our study, on the other hand, reveals equal diagnostic accuracy for STIR and contrasted Dixon. This discrepancy is most likely due to differences in the study design. Costelloe at al. use a semi-quantitative arbitrary conspicuity scale to classify the lesions, whereas in our design the diagnosis is "allor-none" and the statistical analysis is based on binary data. Moreover, Costelloe et al. performed a paired comparison between Dixon and STIR, whereas our design is unpaired with different patients in the Dixon and the STIR group [28].

In an attempt to reduce unnecessary gadolinium application, especially in patients that receive multiple WBMRI as part of surveillance or therapy-response protocols, whole-body diffusion weighted imaging (WB-DWI) with apparent diffusion coefficient maps (ADC) has been suggested as an effective alternative to the contrasted sequences $[29,30]$. Previous studies report a high diagnostic accuracy for WBDWI either as stand-alone [31,32] or as protocol addition to T1w and STIR sequences for the differentiation between malignant and benign lesions [26,33]. In conclusion, there is increasing evidence towards replacement of gadolinium with WB-DWI sequences for the diagnosis of osseous metastasis either for the first diagnostic or in follow up settings.

Our study did not include DWI for technical reasons. In addition, it is limited by an observational retrospective design for a single cohort, deriving from a single center and from the same MRI scanner manufacturer (Philips). However, the high (approximately 100\%) follow-up rate, the low bias rate and the high number of recruited subjects substantially increase the confidence of the results, which qualify for a grade $\mathrm{C}$ or low to moderate recommendation grade according to the Oxford [34] and GRADE criteria for evidence-based medicine $[35,5]$. This result encourages the design of prospective casecontrol studies with data recruitment from different centers and scanners to increase the level of evidence for WB-MRI applications in breast cancer screening.

\section{Conclusion and Clinical Significance}

WB-MRI is a highly sensitive and specific method for the detection of bone metastases and has a promising potential to partially replace the traditional methods of CT-CAP and TC$99 \mathrm{~m}$ bone scan. With WB-MRI, bone metastases can be detected with equal diagnostic accuracy in both field strengths 1.5T and $3 \mathrm{~T}$. This suggests that patients with limited access to or incompatibility with 3T scanners can be investigated in $1.5 \mathrm{~T}$ field strength devices without any drawbacks in the diagnostic accuracy. Application of gadoteridol does not significantly boost the diagnostic accuracy of WB-MRI regarding the detection of osseous metastases, which calls for cautious proofing of the clinical indication and perhaps restricted usage upon demand in the future protocol design.

\section{Disclosures}

The authors confirm that there is no conflict of interest or no financial benefits regarding this manuscript.

\section{References}

1. Wu L-M, Gu H-Y, Zheng J, Xu X, Lin HL, et al. Diagnostic value of wholebody magnetic resonance imaging for bone metastases: a systematic review and meta-analysis. J Magn Reson Imaging. 2011; 34(1): 128-135. doi: $10.1002 / j m r i .22608$

2. O'Sullivan GJ, Carty FL, Cronin CG. Imaging of bone metastasis: An update. World J Radiol. 2015; 7(8): 202-211. doi: 10.4329/wjr.v7.i8.202

3. Heindel W, Gübitz R, Vieth V, Weckesser M, Schober O, Schäfers M. The diagnostic imaging of bone metastases. Dtsch Arzteblatt Int. 2014; 111(44): 741-747. doi: 10.3238/arztebl.2014.0741

4. Peccatori FA, Codacci-Pisanelli G, Del Grande M, Scarfone G, Zugni F, Petralia G. Whole body MRI for systemic staging of breast cancer in pregnant women. Breast. 2017; 35: 177-181. doi: 10.1016/j.breast.2017.07.014

5. Atkins $D$, Best $D$, Briss PA, Eccles $M$, Falck-Ytter $Y$, Flottorp $S$, et al. Grading quality of evidence and strength of recommendations. BMJ. 2004; 328(7454): 1490. doi: 10.1136/bmj.328.7454.1490

6. Houssami N, Costelloe $\mathrm{CM}$. Imaging bone metastases in breast cancer: evidence on comparative test accuracy. Ann Oncol. 2012; 23(4): 834-843. doi: 10.1093/annonc/mdr397

7. Appropriateness Criteria. https://acsearch.acr.org/list 2017.

8. Huynh PT, Lemeshko SV, Mahoney MC, Newell MS, Bailey L, Barke LD. et al. ACR Appropriateness Criteria ${ }^{\circledR}$ Stage I Breast Carcinoma. J Am Coll Radiol. 2016; 13(11): e53-e57. doi: 10.1016/j.jacr.2016.09.024

9. Moy L, Newell MS, Mahoney MC, Bailey L, Barke LD, Carkaci S, et al. ACR Appropriateness Criteria Stage I Breast Cancer: Initial Workup and Surveillance for Local Recurrence and Distant Metastases in Asymptomatic Women. J Am Coll Radiol. 2016; 13(11S): e43-e52. doi: 10.1016/j.jacr.2016.09.023

10. Advanced breast cancer overview - NICE Pathways. https://pathways. nice.org.uk/pathways/advanced-breast-cancer\#content=viewnode\%3Anodes-imaging-assessment 2017.

11. Cardoso F, Costa A, Senkus E, Aapro $M$, André F, Barrios $C H$, et al. 3rd ESO-ESMO International Consensus Guidelines for Advanced Breast Cancer (ABC 3). Ann Oncol. 2017; 28(12): 3111. doi: 10.1093/annonc/ $\mathrm{mdx} 036$

12. Kosmin M, Makris A, Joshi PV, Ah-See ML, Woolf D, Padhani AR. The addition of whole-body magnetic resonance imaging to body computerised tomography alters treatment decisions in patients with metastatic breast cancer. Eur J Cancer. 2017; 77: 109-116. doi: 10.1016/j. ejca.2017.03.001

13. Di Gioia D, Stieber $P$, Schmidt GP, Nagel D, Heinemann V, Baur-Melnyk A. Early detection of metastatic disease in asymptomatic breast cancer patients with whole-body imaging and defined tumour marker increase. Br J Cancer. 2015; 112(5): 809-818. doi: 10.1038/bjc.2015.8

14. Cohen JF, Korevaar DA, Altman DG, Bruns DE, Gatsonis CA, Hooft L, et al. STARD 2015 guidelines for reporting diagnostic accuracy studies: explanation and elaboration. BMJ Open. 2016; 6(11): e012799. doi: 10.1136/bmjopen-2016-012799

15. Cook C, Cleland J, Huijbregts P. Creation and Critique of Studies of Diagnostic Accuracy: Use of the STARD and QUADAS Methodological Quality Assessment Tools. J Man Manip Ther. 2007; 15(2): 93-102. doi: 10.1179/106698107790819945

16. Wood R, Bassett K, Foerster V, Spry C, Tong L. 1.5 Tesla magnetic resonance imaging scanners compared with 3.0 Tesla magnetic resonance imaging scanners: systematic review of clinical effectiveness. CADTH Technol Overv. 2012; 2(2): e2201.

17. Ramalho $M$, Herédia V, Tsurusaki M, Altun E, Semelka RC. Quantitative and qualitative comparison of 1.5 and 3.0 Tesla MRI in patients with chronic liver diseases. J Magn Reson Imaging. 2009; 29(4): 869-879. doi: 10.1002/jmri.21719

18. Wood R, Bassett $K$, Foerster V, Spry C, Tong L. PROS AND CONS OF 1.5 T MRI VERSUS 3.0 T MRI. Canadian Agency for Drugs and Technologies in Health. 2011. 
19. Šimundić AM. Measures of Diagnostic Accuracy: Basic Definitions. EJIFCC. 2009; 19(4): 203-211.

20. Suva LI, Griffin RJ, Makhoul I. Mechanisms of bone metastases of breast cancer. Endocr Relat Cancer. 2009; 16(3): 703-713. doi: 10.1677/ERC-090012

21. Lenkinski RE. Gadolinium Retention and Deposition Revisited: How the Chemical Properties of Gadolinium-based Contrast Agents and the Use of Animal Models Inform Us about the Behavior of These Agents in the Human Brain. Radiology. 2017; 285(3): 721-724. doi: 10.1148/radiol.2017172138

22. Kanda $T$, Nakai $Y$, Hagiwara A, Oba $H$, Toyoda K, Furui S. Distribution and chemical forms of gadolinium in the brain: a review. Br J Radiol. 2017; 90(1079). doi: 10.1259/bjr.20170115

23. Gulani V, Calamante F, Shellock FG, Kanal E, Reeder SB, et al. Gadolinium deposition in the brain: summary of evidence and recommendations. Lancet Neurol. 2017; 16(7): 564-570. doi: 10.1016/S1474-4422(17)30158-8

24. Walker R, Kessar P, Blanchard R, Dimasi M, Harper K, DeCarvalho V, et al. Turbo STIR magnetic resonance imaging as a whole-body screening tool for metastases in patients with breast carcinoma: preliminary clinical experience. J Magn Reson Imaging. 2000; 11(4): 343-350.

25. Pearce $T$, Philip $S$, Brown J, Koh DM, Burn PR. Bone metastases from prostate, breast and multiple myeloma: differences in lesion conspicuity at short-tau inversion recovery and diffusion-weighted MRI. Br J Radiol. 2012; 85(1016): 1102-1106. doi: 10.1259/bjr/30649204

26. Jacobs MA, Macura KJ, Zaheer A, Antonarakis ES, Stearns V, Wolff AC, et al. Multiparametric Whole-body MRI with Diffusion-weighted Imaging and ADC Mapping for the Identification of Visceral and Osseous Metastases From Solid Tumors. Acad Radiol. 2018. doi: 10.1016/j.acra.2018.02.010

27. Thomson V, Pialat J-B, Gay F, Coulon A, Voloch A, Granier A, et al. Whole-body MRI for metastases screening: a preliminary study using $3 D$ VIBE sequences with automatic subtraction between noncontrast and contrast enhanced images. Am J Clin Oncol. 2008; 31(3): 285-292. doi: 10.1097/COC.0b013e31815e3ff4
28. Costelloe CM, Madewell JE, Kundra V, Harrell RK, Bassett RL, Ma J. Conspicuity of bone metastases on fast Dixon-based multisequence whole-body MRI: clinical utility per sequence. Magn Reson Imaging. 2013; 31(5): 669-675. doi: 10.1016/j.mri.2012.10.017

29. Padhani AR, Makris A, Gall P, Collins DJ, Tunariu N, de Bono JS. Therapy monitoring of skeletal metastases with whole-body diffusion MRI. J Magn Reson Imaging. 2014; 39(5): 1049-1078. doi: 10.1002/jmri.24548

30. Woolf DK, Padhani AR, Makris A. Assessing response to treatment of bone metastases from breast cancer: what should be the standard of care? Ann Oncol. 2015; 26(6): 1048-1057. doi: 10.1093/annonc/mdu558

31. Nakanishi $K$, Kobayashi $M$, Nakaguchi K, Kyakuno $M$, Hashimoto $N$, Onishi $\mathrm{H}$, et al. Whole-body MRI for detecting metastatic bone tumor: diagnostic value of diffusion-weighted images. Magn Reson Med Sci. 2007; 6(3): 147-155.

32. Gandage $S$, Kachewar $S$, Aironi V, Nagapurkar A. A comparative study of whole body DWIBS MRI versus bone scan for evaluating skeletal metastases. Australas Med J. 2012; 5(12): 619-622. doi: 10.4066/ AMJ.2012.1500

33. Winfield JM, Poillucci G, Blackledge MD, Collins DJ, Shah V, Tunariu N, et al. Apparent diffusion coefficient of vertebral haemangiomas allows differentiation from malignant focal deposits in whole-body diffusionweighted MRI. Eur Radiol. 2018; 28(4): 1687-1691. doi: 10.1007/s00330017-5079-2

34. Oxford Centre for Evidence-based Medicine - Levels of Evidence. 2009 https://www.cebm.net/2009/06/oxford-centre-evidence-basedmedicine-levels-evidence-march-2009 Accessed April 12, 2018.

35. Holger Schünemann, Jan Brożek, Gordon Guyatt, Andrew Oxman. GRADE handbook. http://gdt.guidelinedevelopment.org/app/handbook/handbook.html Accessed April 12, 2018. 